\title{
In-situ TEM Observation of Alloy Phase Formation in Isolated Nanometer-sized Particles in the Pb-Sn System
}

\author{
H. Mori*, J.-G. Lee* and H. Yasuda** \\ *Research Center for Ultra-High Voltage Electron Microscopy, Osaka University, Yamadaoka, Suita, \\ Osaka 565-0871, Japan \\ **Department of Mechanical Engineering, Kobe University, Rokkodai, Nada, Kobe 657-8501, \\ Japan
}

It is now well established that nanometer-sized materials exhibit electronic, magnetic, optic, and thermal properties which are significantly different from those of the corresponding bulk materials. How to create new properties by manipulating materials in the nanometer range is one of current scientific and technological issues. Along with this trend, there is an urgent need for the phase diagram of nanometer-sized alloy systems to extend the usage of new functional nanometer-sized particles in such fields as nanoelectronics and catalysis. However, so far studies have been mainly concerned with nanometer-sized pure substances and studies on nanometer-sized alloy particles are quite limited [1-4]. To get fundamental understanding the phase equilibrium of nanometer-sized alloy particles, studies on the solid-solibility in nanometer-sized alloy are significantly important. To the authors' knowledge no quantitative experimental analysis on the solid solubility in nanometer-sized alloy particles has been reported. Based upon this premise, the present paper describes the size effect on the alloy phase formation and the solid solubility in the Pb-Sn binary system over a temperature range from $\mathrm{RT}$ to $130^{\circ} \mathrm{C}$.

Preparation of nanometer-sized lead particles and subsequent vapor-deposition of tin onto lead particles were carried out using a double-source evaporator installed in the specimen chamber of a Hitachi H-800 type 200kV TEM. The evaporator consisted of two spiral-shaped tungsten filaments. An amorphous carbon film mounted on a molybdenum grid was used as a supporting film. A heating holder was employed to control the temperature of the amorphous carbon film. Using this evaporator, lead was first evaporated from one filament heated by the Joule effect onto the supporting film kept at certain temperatures $\left(\mathrm{RT}, 110^{\circ} \mathrm{C}, 130^{\circ} \mathrm{C}\right)$, and nanometer-sized lead particles were produced on the film. Next, tin was evaporated from the other filament onto the same film kept at the same temperature. Alloy phase formation in the nanometer-sized particles associated with tin deposition was studied by both bright-field images (BFIs) and selected area electron diffraction patterns (SAEDs). Subsequent to the study on alloy phase formation at $110^{\circ} \mathrm{C}$, phase alterations in particles associated with cooling from $110^{\circ} \mathrm{C}$ to room temperature (RT) and with re-heating from RT to $110^{\circ} \mathrm{C}$ were also examined. The microscope was equipped with a turbo-molecular pumping system to achieve a base pressure of around $3 \times 10^{-5} \mathrm{~Pa}$ in the specimen chamber. The chemical composition of alloy particles was analyzed by energy dispersive X-ray spectroscopy (EDS).

In approximately $16-\mathrm{nm}$-sized alloy particles kept at $130^{\circ} \mathrm{C}$, the formation of a liquid phase which is not an equilibrium phase in bulk at $130^{\circ} \mathrm{C}$, was confirmed. At $110^{\circ} \mathrm{C}$, however, these particles exhibited a microstructure of a mixture of two crystalline phases at compositions near the eutectic composition. In view of the fact that the eutectic temperature of bulk alloys is $183^{\circ} \mathrm{C}$, it seems safe to consider that suppression of the eutectic temperature as much as $50^{\circ} \mathrm{C}$ took place in association 
with the particle-size reduction. In approximately 16-nm-sized lead solid solution, the tin solubility was higher than 56 atomic percent at $110^{\circ} \mathrm{C}$, which is almost five times higher than that of the corresponding bulk materials. Even at room temperature the tin solubility amounted to approximately 30 atomic percent. Such an enhancement of atomic solubility was confirmed also in the tin-rich side. In addition, it was revealed that the temperature dependence of lattice parameter in nanometer-sized lead particles is quite different from that of the corresponding bulk materials. Based upon these experimental results, phase equilibrium of nanometer-sized alloy particles in the $\mathrm{Pb}-\mathrm{Sn}$ system will be discussed.

\section{References}

[1] J. -G. Lee et al., Phys. Rev. B 65 (2002) 132106.

[2] J. -G. Lee et al., Phys. Rev. B 66 (2002) 012105.

[3] J. -G. Lee and H. Mori, Phil. Mag. 84 (2004) 2675.

[4] J. -G. Lee and H. Mori, Phys. Rev. B 70 (2004) 144105.

[5] J. -G. Lee and H. Mori, Phys. Rev. Lett. 93 (2004) 235501.

[6] This work was supported by "Priority Assistance of the Formation of Worldwide Renowned Centers of Research - The 21st Century COE Program (Project: Center of Excellence for Advanced Structural and Functional Materials Design)" from the Ministry of Education, Sports, Culture, Science and Technology of Japan. A part of the present work was supported by the Ministry of Education, Sports, Culture, Science and Technology of Japan under a Grant-in-Aid for Scientific Research (Grant No. 15074213 and 15206070).

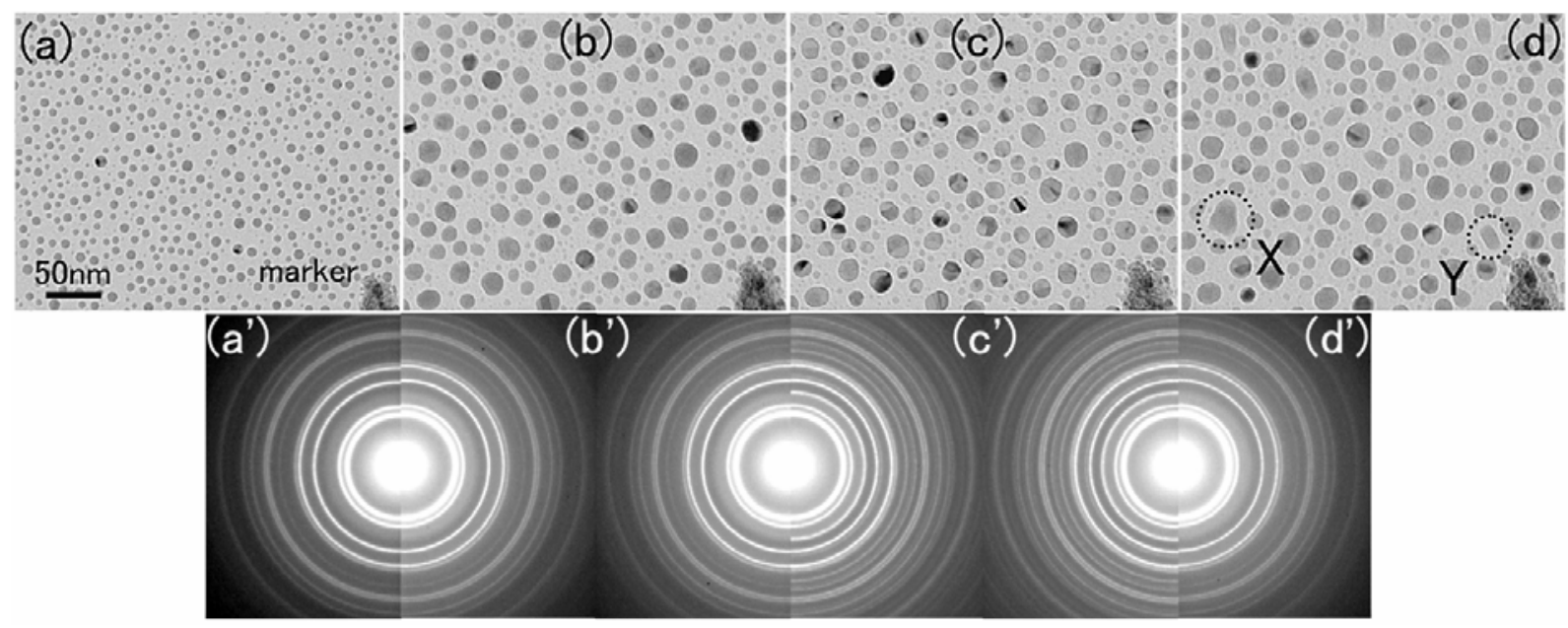

Fig.1 A series of electron micrographs showing alloy phase formation in nanometer-sized particles in the $\mathrm{Pb}-\mathrm{Sn}$ system. (a) as-produced lead particles on an amorphous carbon film kept at $110^{\circ} \mathrm{C}$, (b) the same area as (a) after tin deposition, (c) the same area as (b) after cooled down to RT, (d) the same area as (c) after re-heating up to $110^{\circ} \mathrm{C}$. (a'), (b'), (c'), and (d') SAEDs corresponding to (a), (b), (c), and (d), respectively. A small dirt at the bottom-right corner of individual BFIs serves as a marker showing a fixed position. It was revealed by EDS that particles shown in (b) contained 56 atomic percent tin, on average, which is almost five times higher than the solubility limit of tin in the bulk lead-tin solid solution at the same temperature (i.e., 10 at.\%) [5]. 